\title{
Civilisations
}

Revue internationale d'anthropologie et de sciences

humaines

49 | 2002

Pain, fours et foyers des temps passés

\section{Les foyers et les fours domestiques en Egée au Néolithique et à l'Age du Bronze}

\section{Sandra Prevost-Dermarkar}

\section{OpenEdition \\ Journals}

Édition électronique

URL : http://journals.openedition.org/civilisations/1475

DOI : 10.4000/civilisations. 1475

ISSN : 2032-0442

\section{Éditeur}

Institut de sociologie de l'Université Libre de Bruxelles

\section{Édition imprimée}

Date de publication : 3 juin 2002

Pagination : 223-237

ISBN : 0009-8140

ISSN : 0009-8140

Référence électronique

Sandra Prevost-Dermarkar, « Les foyers et les fours domestiques en Egée au Néolithique et à l'Age du Bronze », Civilisations [En ligne], 49 | 2002, mis en ligne le 01 juin 2005, consulté le 01 mai 2019. URL http://journals.openedition.org/civilisations/1475; DOI : 10.4000/civilisations. 1475

Ce document a été généré automatiquement le 1 mai 2019.

(c) Tous droits réservés 


\title{
Les foyers et les fours domestiques en Egée au Néolithique et à l'Age du Bronze
}

\author{
Sandra Prevost-Dermarkar
}

\section{Introduction}

1 L'étude proposée ici a été stimulée par l'absence de travaux de synthèse et d'intérêt porté aux dispositifs de combustion retrouvés en Grèce (essentiellement en Grèce du nord, en Grèce centrale, dans le Péloponnèse, en Crète et dans les Cyclades) et dans le sud des Balkans (Albanie, Macédoine ex-yougoslave et Bulgarie ; Deshayes, 1974, p.67-91)1. Elle se limite chronologiquement au Néolithique (de 7000 à 3250 BC environ) et à l'Age du Bronze (de 3250 à $1050 \mathrm{BC}$ environ) ${ }^{2}$. Elle ne prend en compte que les structures s'inscrivant dans un cadre domestique, excluant ainsi les autres fours (céramique, chaux et métallurgie).

2 Cette étude se fonde essentiellement sur les vestiges archéologiques, même si malheureusement ces structures ne sont pas souvent décrites en détail dans les publications. Pour essayer de pallier le manque d'informations, des exemples ethnologiques et expérimentaux seront proposés à titre de comparaison. Mais ces exemples ne serviront qu'à comprendre des caractéristiques techniques et fonctionnelles identifiées d'abord sur les vestiges archéologiques.

3 Les structures de combustion ont d'abord fait l'objet d'une analyse morphologique qui a permis d'établir une typologie. Il a paru ensuite intéressant d'essayer de comprendre leur mode de fonctionnement et de suggérer quelques fonctions 


\section{Analyse morphologique}

\subsection{Les foyers}

4 distinguer plusieurs types selon leur mode de construction : les foyers à plat, les foyers creusés et les foyers surélevés.

\subsubsection{Les foyers à plat}

5 Ce type a été défini comme un foyer établi directement sur un sol sans aménagement préalable (Leroi-Gourhan, 1979, p.10)3. Cette définition est trop restrictive pour notre documentation, car la plupart des exemples étudiés sont caractérisés par un petit aménagement préalable afin d'isoler le feu du sol. Cependant, la surélévation qui en résulte est très faible et ne traduit pas une volonté réelle de surhaussement comme dans le cas des foyers surélevés; on ne peut donc pas confondre nos foyers à plat et nos foyers surélevés.

6

Dans le cas des foyers à plat simples, du combustible a été déposé sur le sol, sans délimitation préalable, puis brûlé (Treuil, 1992, p.45). Leur plan reste souvent inconnu ou irrégulier du fait de leur improvisation. Ils sont reconnus uniquement par la surface du sol rubéfiée (noircie ou rougie) par le feu. En général, les descriptions de ces foyers sont très succinctes et l'identification elle-même est très difficile, surtout lorsque l'habitation a subi un incendie.

7 Les foyers à plat aménagés correspondent aux foyers les plus couramment utilisés dans l'ensemble du monde égéen et leur usage augmente à l'Age du Bronze. Ils sont parfois entourés d'une bordure en pierre (Goldman, 1932, p.14) ou en terre à bâtir ${ }^{4}$ (un exemple à Kastanas, où la bordure est constituée de briques crues, Fig. 1 ; Hänsel, 1979, p.183) se présentant sous la forme d'un petit muret. Dans de nombreux exemples, la surface de leur zone de combustion a reçu un revêtement se constituant d'un seul ou de plusieurs matériaux : un lit de cailloux (Ridley et Wardle, 1979, p.197) ou de tessons placés à la surface du foyer (Renfrew et al., 1986, p.205) ou bien encore une simple couche de terre à bâtir (Aslanis, 1985, p.60). Dans la plupart des cas, les foyers présentent un plan circulaire d'un mètre de diamètre environ ou une surface rectangulaire d'environ un mètre carré. 
Fig. 1 : Foyer à plat (d'un diamètre $d^{\prime} 1 \mathrm{~m} 30$ ) avec revêtement en terre à bâtir et bordure en briques crues de Kastanas (HÄNSEL, B. 1979, p. 182)

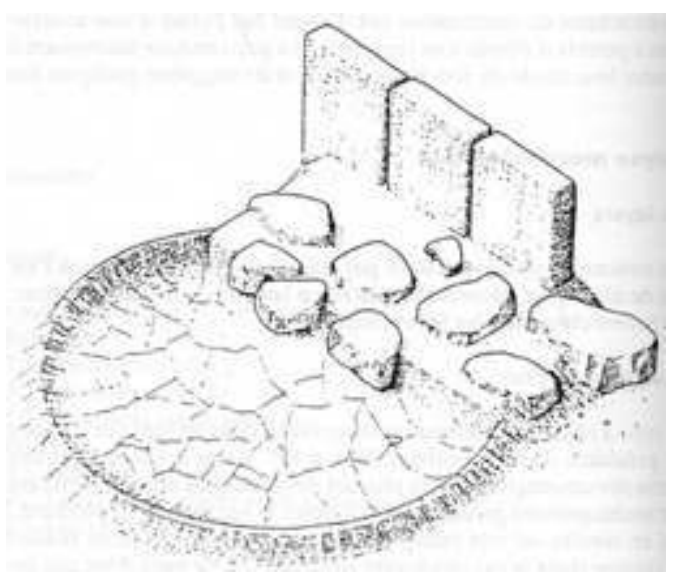

\subsubsection{Les foyers creusés}

Couramment employé depuis le Paléolithique, ce type de foyers a un fonctionnement des plus simples car le combustible est posé directement dans une cuvette creusée dans le sol. Tous les exemplaires de foyers creusés ont un plan plus ou moins circulaire correspondant à la forme que prend naturellement une excavation pratiquée dans le sol avec des moyens rudimentaires. Le diamètre à l'ouverture varie de $0,30 \mathrm{~m}$ à $1,00 \mathrm{~m}$.

Une première distinction peut être pratiquée entre les foyers creusés en cuvette et ceux creusés en fosse.

Les foyers en cuvette sont définis comme des dispositifs " établis dans une dépression creusée aux parois en pente douce» (C. Perles, dans Leroi-Gourhan, 1979, p.48). Il en existe de deux types, simple ou aménagé. Le premier type correspond à ceux dont la dépression est simplement creusée dans le sol de l'habitat ou de la cour sans aménagement secondaire. Les exemples retrouvés de ce type sont trop peu nombreux pour en déduire une répartition géographique et une évolution chronologique caractéristiques. Le second type traduit «l'existence d'un acte constructif supplémentaire, s'ajoutant à l'acte primaire de creusement " (Molist, 1986, p.25). Il se caractérise par des aménagements : la bordure et/ou le revêtement du fond et des parois de la dépression. La bordure constituée d'une rangée de pierres (Gimbutas et al., 1989, p.36) ou bien façonnée en terre à bâtir est disposée sur toute la périphérie de l'ouverture de la dépression. Le revêtement en terre à bâtir est utilisé comme un enduit qui est étalé sur toute la surface de la cuvette (Fig. 2) (Gimbutas et al., 1989, p.59). Il semble que ce type soit plus employé au Néolithique qu'à l'Age du Bronze.

Fig. 2 : Foyer creusé en cuvette avec revêtement de terre (GIMBUTAS, M. \& al. 1989, p.59).

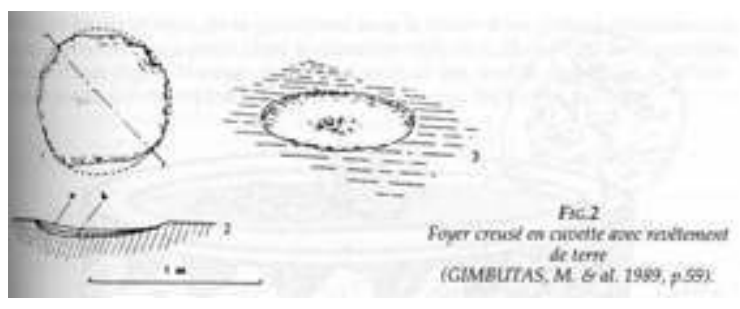


11 Les foyers en fosse sont des foyers aménagés dans une dépression dont les parois sont "verticales ou subverticales » (C. Perles dans Leroi-Gourhan, 1979, p.48) et dont la profondeur varie entre 0,45 et $0,80 \mathrm{~m}$. A l'instar des foyers en cuvette, ils présentent les types simple et aménagé (Fig. 3 et 4) (Efstratiou, 1985, p.21).

Fig. 3 : Foyer creusé en fosse avec revêtement de pierre (GIMBUTAS, M. \& al. 1989, p.54).

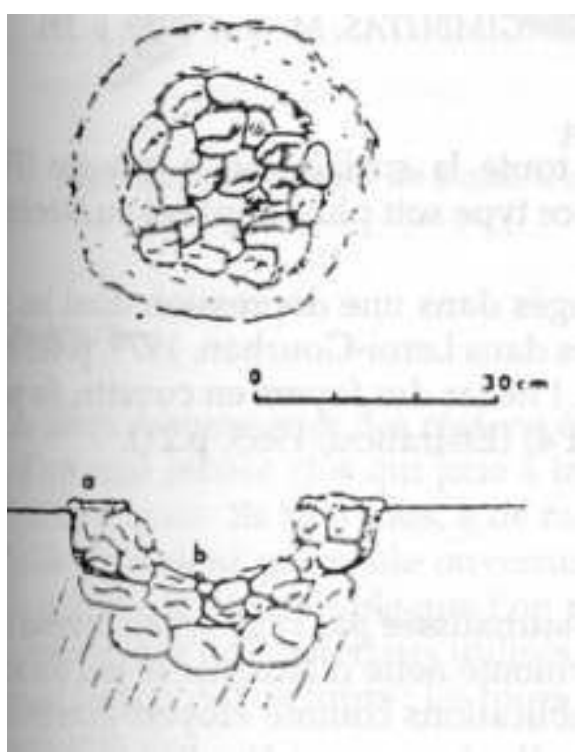

Fig. 4 : Foyer creusé avec bordure en terre à bâtir d'Achilléion (GIMBUTAS, M. \& al. 1989, p.39)

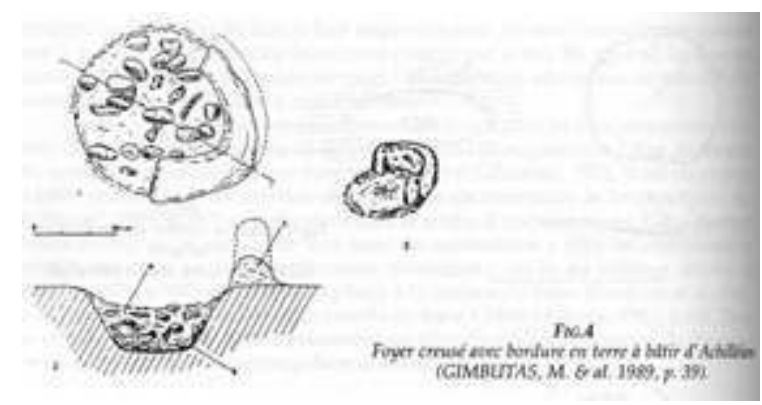

\subsubsection{Les foyers surélevés}

La zone de combustion est nettement surhaussée par rapport au niveau du sol. En effet, cette surélévation résulte d'une volonté nette d'éloigner le feu du sol. Ces foyers sont souvent désignés dans les publications comme "foyers-autels", terme lié à leur fonction supposée. Les foyers surélevés peuvent être fixes sur une plate-forme ou mobiles.

Les foyers fixes sont établis sur une plate-forme de hauteur très variable. Ils sont essentiellement de deux formes: circulaires (le foyer de Pylos atteignant 4,00 m de diamètre ; Blegen et Rawson, 1966, p.85-87) ou rectangulaires avec des longueurs variant de $0,50 \mathrm{~m}$ à $1,90 \mathrm{~m}$ (exemple du foyer de Malia d'épaisseur environ 0,40 $\mathrm{m}$; Demargne, 1928, p.19). La plate-forme est constituée de pierres agencées face plane vers le haut, ou bien d'une seule grande dalle de pierre épaisse (deux exemples de ce type à Malthi : leurs dalles sont épaisses de 0,40 m.; Valmin, 1938, p.128). Elle peut aussi être construite en terre à bâtir sous la forme d'une épaisse couche ou combiner plusieurs matériaux de 
nature différente : un lit de pierres ou de tessons en fondation recouvert d'une couche de terre à bâtir. Certaines plates-formes enduites comportent une légère dépression sous la forme d'une petite cuvette entourée d'un renflement. Ces foyers de l'Age du Bronze peints ou incisés ont été retrouvés dans les palais du Péloponnèse et de Crète.

Les foyers surélevés mobiles sont souvent nommés "foyers-tripodes». Ils ont tous été retrouvés en Crète en contexte palatial et datent du MM I ou du MM III ${ }^{5}$. Fabriqués en céramique, ils se présentent sous la forme d'un plateau circulaire peu profond, posé sur trois pieds, dont le diamètre varie de $0,28 \mathrm{~m}$ à $0,90 \mathrm{~m}$. Il peut être entouré d'une légère bordure présentant parfois des motifs de vagues et d'astérisques peints (un exemple à Cnossos, Fig. 5) (Evans, 1921-1936, p.179).

Fig. 5 : Foyer surélevé mobile d'un diamètre de 40 cm (EVANS, A. 1921-1936, p.180).

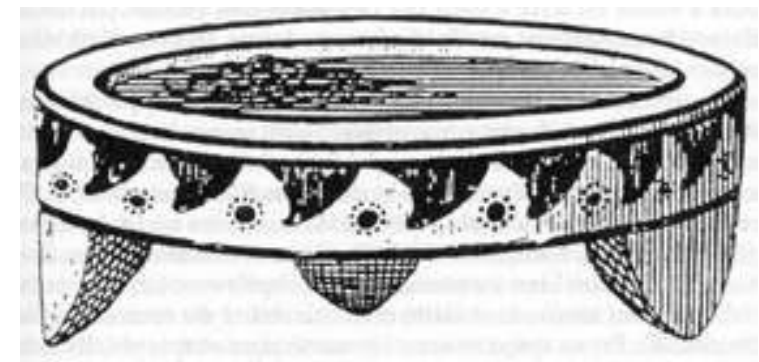

\subsection{Les fours}

15 Les fours domestiques des régions égéennes et balkaniques sont toujours constitués d'un seul espace clos qui joue à la fois le rôle de chambre de combustion et de chambre à cuire. Ils sont tous, à de rares exceptions près, d'accès frontal c'est-à-dire qu'ils possèdent une seule ouverture située à la base de la voûte (Fig. 6). Ce type de four est le plus simple que l'on puisse rencontrer.

Fig. 6 : Reconstitution hypothétique du four 1 de Dikili Tash (DESHAYES, J. 1974, p.72)

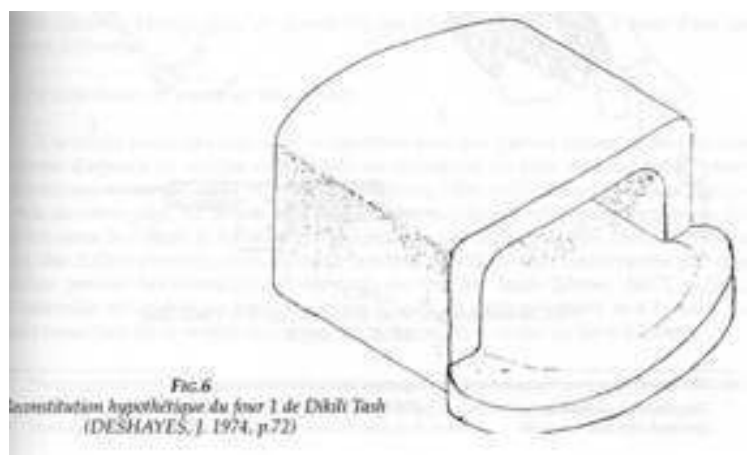

L'observation des matériaux utilisés pour la construction de la voûte permet de distinguer trois types de fours: les fours en terre, les fours de pierre et de tessons et les «fours-pithoi ». 


\subsubsection{Les fours en terre}

17 Les fours de ce type sont les plus fréquents. En effet, la terre argileuse est un matériau plastique très facile à trouver (il existe en abondance dans la nature). Aisé à travailler, il présente en outre des propriétés thermiques intéressantes ${ }^{6}$.

Les fours à voûte en terre à bâtir ont des plans très variés : circulaires, ovales, semicirculaires, trapézoïdaux, carrés et rectangulaires. Ils ont sensiblement tous la même superficie (environ $1,00 \mathrm{~m}^{2}$ ).

Les découvertes archéologiques nous indiquent que ce type de four était toujours construit de la même façon : un soubassement, une sole et une voûte.

Le soubassement, qui forme la base du four, est constitué le plus souvent de plusieurs couches de pierres, de cailloux et de galets (Georgiev, 1963, p.175). Parfois un simple cailloutis (Georgiev et al., 1986, p.114) ou même un lit de tessons posés à plat (Treuil, 1992, p.46-47) suffisent (Fig. 7). Ce soubassement peut être aménagé dans une fosse creusée ou bien au niveau du sol, surélevant ainsi toute la structure. Un bon soubassement assure la solidité et la durabilité du four en l'isolant du sol, donc de l'humidité. En ce qui concerne les matériaux employés, ils conservent la chaleur pour la restituer ensuite progressivement.

21 La sole qui recouvre le soubassement se définit comme une surface plane sur laquelle les combustibles se consument et les aliments à cuire sont posés. Elle est constituée d'une couche de terre très argileuse qui comporte beaucoup de dégraissants ${ }^{7}$ l'empêchant de se fissurer sous l'effet de la chaleur (Gimbutas et al., 1989, p.39). Cette couche est lissée à la main comme en témoignent les traces de doigts que l'on retrouve parfois sur les fragments archéologiques. Une plage avant, souvent aménagée en même temps que le soubassement, reçoit aussi cette couche de terre argileuse dont l'épaisseur varie entre $2 \mathrm{~cm}$ et $8 \mathrm{~cm}$.

Fig. 7 : Plan du soubassement et de la sole du four 8 de Dikili Tash (TREUIL, R. 1992)

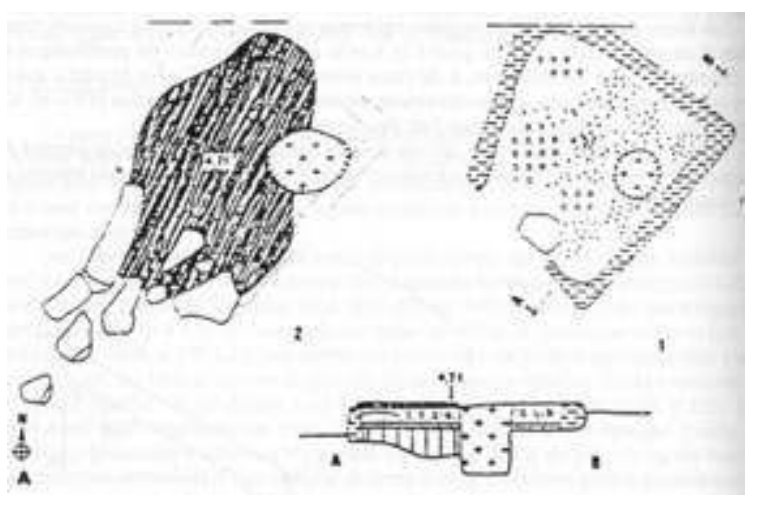

Les parois et la voûte recouvrant l'ensemble sont construites en terre à bâtir. Plusieurs techniques sont identifiables:

- celle utilisant une armature en bois8. Cette technique est attestée archéologiquement par les empreintes laissées par les poteaux et les branches plantées dans le soubassement du four (Georgiev, 1981, p.73). Elle utilise une forme en vannerie de branchages recouverte de terre à bâtir lissée à la main. 
- celle du colombin. Cette technique, proche de celle du potier, semble être la plus courante. L'artisan réalise des colombins de terre à bâtir qu'il monte ensuite les uns au-dessus des autres, formant ainsi progressivement de bas en haut les parois et la voûte du four (Darcque et al., 1992, p.715) Cette technique est toujours employée actuellement en Grèce.

- celle utilisant une forme de sable ou de terre. Cette technique, difficilement identifiable archéologiquement, consiste à entasser sur la sole de la terre ou du sable, puis à façonner ce tas afin qu'il serve de moule à la voûte et qu'il lui donne sa forme. La «bouche » du four est délimitée et aménagée par un coffrage de bois. Ensuite, une ou plusieurs couches de terre à bâtir sont appliquées sur ce moule. Après quelques jours de séchage, le sable ou la terre sont retirés par la « bouche » du four. Il faut alors rectifier à l'aide d'un gros galet ou simplement avec la paume de la main les parois tant à l'intérieur qu'à l'extérieur.

La forme et les dimensions de la voûte ne sont pas connues avec certitude, car celle-ci n'est jamais conservée dans sa totalité. Il semblerait toutefois qu'elle ait été le plus souvent en berceau et parfois surbaissée. La largeur de l'ouverture, souvent mesurable, oscille entre 0,30 et $0,40 \mathrm{~m}$.

Les exemples ethnologiques montrent qu'après la construction, le four est laissé à sécher. Si des fissures apparaissent, elles doivent être colmatées par compression et rajout de terre à bâtir. Le four sèche ensuite pendant environ quinze jours; au bout de ce laps de temps, de petits feux de plus en plus intenses sont régulièrement allumés. Finalement, un grand feu est allumé afin de figer le four dans sa forme définitive.

\subsubsection{Les fours de pierre et de tessons}

Certaines parois de four sont construites avec des pierres plates et parfois des tessons disposés en cercles superposés de diamètres de plus en plus petits pour obtenir une voûte en encorbellement ${ }^{9}$ (Tsountas, 1908, p.52). Ils sont ensuite recouverts de terre plus ou moins argileuse Cette technique est encore employée en Grèce dans le village d'Achilléion (Gimbutas et al., 1974, p.72-73). Dans quelques cas, des dalles dressées verticalement bordent la sole et sont recouvertes par des dalles posées horizontalement servant de toit au four (Shaw, 1977, p.226). L'ensemble est enduit de terre argileuse. Quant au soubassement et à la sole, ils sont construits de la même façon que ceux des fours à voûte en terre à bâtir.

\subsubsection{Les « fours-pithoi »}

Ces structures de combustion très particulières ne sont attestées qu'en contexte archéologique (de nombreux exemples proviennent du site de Thermi sur l'île de Lesbos au Bronze Ancien; Lamb, 1936, p.59). C'est le corps ovale de ces très grands vases (d'environ $1 \mathrm{~m}$ de diamètre et de $2 \mathrm{~m}$ de haut) en argile grossière qui joue le rôle de la chambre à cuire. La cuisson se faisait peut-être de façon indirecte par induction de la chaleur à partir des parois en argile ${ }^{10}$. Ces pithoi peuvent être enfoncés horizontalement dans le sol et une plate-forme de terre brûlée et durcie s'étend alors devant leur ouverture, formant une sorte de plage avant. Ils peuvent aussi être enfoncés verticalement dans le sol, l'ouverture vers le haut ${ }^{11}$. 


\section{Mode de fonctionnement}

27 Le choix des matériaux et l'aménagement des dispositifs de combustion dépendent étroitement de leur fonctionnement. Les deux types de structures étudiées ne fonctionnent pas de la même façon : les foyers sont des dispositifs à combustion ouverte, tandis que les fours sont des structures à combustion couverte.

\subsection{Les foyers}

28 Les hommes préhistoriques avaient à leur disposition une gamme de combustibles très variée. Ces derniers sont parfois identifiables en fouille, par les traces qu'ils ont laissées. Appelés produits de combustion, il s'agit essentiellement de charbons et de cendres. Ces restes témoignent de l'utilisation du bois comme combustible. De nombreux autres matériaux tels que les os, la graisse animale, la moelle osseuse, la tourbe ou les excréments d'animaux ont pu être utilisés. Cependant, les traces laissées sont le plus souvent difficiles à identifier.

D'une manière générale, l'allumage d'un feu dans un foyer est très simple. Les combustibles sont placés en tas au milieu de la structure. Ils ne doivent pas être tassés afin de permettre une bonne oxygénation de la combustion. Ceux de petite taille doivent être placés à la base et allumés en premier. Le feu est ensuite alimenté en gros combustibles. Le souffle humain suffit à le ventiler et à le ranimer.

30 Selon la taille du foyer et son aménagement ainsi que l'utilisation envisagée, la combustion se fait sous la forme de flammes ou bien de braises. La bordure et le revêtement souvent observés sur les foyers creusés ou à plat permettent de mieux accumuler la chaleur, de protéger des courants d'air et d'éviter l'éparpillement des braises, limitant ainsi les risques d'incendie.

31 Lorsque le revêtement de terre de la zone de combustion est trop craquelé, son efficacité thermique diminue. Il reçoit alors une nouvelle couche de terre à bâtir. Les pierres qui constituent parfois le revêtement et la bordure d'un foyer sont souvent fracturées par un feu intensif et répété. Elles sont alors remplacées, car elles sont devenues trop petites pour continuer à jouer leur rôle calorifère.

32 Le foyer utilisé quotidiennement est rapidement rempli de déchets. Il est nécessaire pour son bon fonctionnement que sa zone de combustion soit régulièrement nettoyée par balayage.

\subsection{Les fours}

Les fours égéens ne possèdent qu'un seul espace destiné à la combustion et à la cuisson. Les deux opérations se font donc successivement. Le chauffage d'un four est une opération très délicate qui doit être menée avec beaucoup de soin. Elle doit permettre d'élever progressivement la température à l'intérieur de la chambre à cuire afin que les parois et la sole accumulent un maximum de chaleur qu'elles restituent ensuite ${ }^{12}$.

34 A partir des expérimentations réalisées (Werner, 1991, p.210-213) et des exemples ethnographiques ${ }^{13}$, il est possible de se faire une idée des différentes étapes de fonctionnement : le chauffage puis le refroidissement du four. 

Certaines activités humaines nécessitant l'énergie du feu laissent des témoignages archéologiques à l'intérieur et tout autour des structures. Il s'agit de les reconnaitre et de les interpréter correctement. Malheureusement, d'autres utilisations ne laissent pas de traces matérielles. Elles ne sont que suggérées par des observations ethnographiques. La présence de plusieurs structures (le plus souvent des foyers) dans et autour d'une même habitation (Gimbutas et al., 1989, p.50-53) suggère des fonctions diverses et peut-être spécifiques à certaines structures. Il est possible de proposer cinq utilisations principales.

\subsection{La cuisson des aliments}

Les déchets alimentaires et les ustensiles de cuisine (du type marmite) retrouvés dans et aux abords des structures peuvent témoigner de cette fonction. L'ensemble de la documentation montre une intégration indiscutable de ces structures dans des espaces domestiques à vocation globalement alimentaire. Elles sont régulièrement associées à des 
dispositifs de stockage sous la forme de silos en argile ou de vases de stockage qui contiennent des graines, des figues ou des olives carbonisées. En dépit de la difficulté à déterminer avec certitude tant les aliments que les procédés employés pour cuire, des études (Perles, 1975, 1977; Julien, 1987, p.31-41) ont montré que bon nombre de techniques de cuisson peuvent être envisagées dès le Paléolithique. Deux grands modes de cuisson sont pratiqués: la cuisson directe pour laquelle les aliments sont soumis directement à l'action de la flamme au moyen d'une broche ou d'une grille, et la cuisson indirecte durant laquelle les aliments sont cuits, déposés dans un récipient ou sur une pierre afin de répartir les effets du feu. Dans les fours (inventés au Néolithique), la cuisson se fait de façon indirecte par réverbération de la chaleur sur les parois. L'utilisation de ces dispositifs permettait sans aucun doute de griller, rôtir, bouillir, étuver, cuire à la vapeur et frire les aliments. La nécessité de cuissons différentes selon les aliments et les "recettes» expliquent sans doute la présence fréquente de foyers à proximité des fours, parfois même accolés à eux (Hänsel, 1979, p.183-184).

\subsection{L'éclairage et le chauffage}

41 Le foyer de cuisine émet de la lumière de façon plus ou moins intense. Ce type d'éclairage peut être complété au besoin par des lampes à huile ou des torches. Le feu du foyer produit nécessairement de la chaleur. Toutefois en dehors d'un apport plus ou moins important de combustible, on connaît peu de choses du contrôle exercé par les hommes préhistoriques sur le rayonnement thermique de combustion. On sait simplement que les pierres du foyer jouent le double rôle d'accumulateur et de libérateur progressif de chaleur. Il en va de même pour les fours qui ressemblent à de grands calorifères. Pendant la chauffe, le soubassement de pierres, la sole de terre, souvent très argileuse et les parois de terre à bâtir emmagasinent un maximum de chaleur. Au moment du refroidissement, cette dernière est libérée pendant des heures (quatre ou cinq) dans toute la pièce.

\subsection{Une fonction sociale}

42 Les structures de combustion reçoivent une place privilégiée dans l'habitation. Le foyer se situe, dans de nombreux cas, au centre de la pièce principale et le four est souvent construit près du mur du fond, face à l'entrée. Ces dispositifs sont des pôles attractifs autour desquels les hommes vivent. L'étude des sociétés traditionnelles montre que dans bien des cas le foyer se charge en plus d'une valeur symbolique.

\subsection{Autres fonctions}

Il est plus que probable que les foyers ont également joué un rôle dans l'élimination des déchets par combustion, comme en témoignent les vestiges alimentaires retrouvés à l'intérieur.

Des activités de fabrication domestiques utilisent l'énergie thermique du feu. Ainsi, la taille du silex, le façonnage de l'os, le travail des peaux, des fibres de toutes sortes, la transformation des matières colorantes (calcination de l'ocre, par exemple) nécessitent l'usage du feu. Certains foyers creusés de très grande taille (environ $4 \mathrm{~m}$ de diamètre), situés dans la cour (Gimbutas et al., 1989, p.53 et 58) et entourés de débris de vases ont pu servir à cuire des poteries ${ }^{14}$. 

pu remplir plusieurs de ces fonctions en même temps ou bien être réservée à une fonction exclusive. Il est dommage que les fouilleurs ne prêtent pas plus d'attention à ces dispositifs, aux indices relatifs à leur(s) utilisation(s), à leur contenu et à ce qui les entoure; cela permettrait sans doute de confirmer certaines fonctions actuellement supposées.

\section{Conclusion}

Au terme de cette étude, et bien que la documentation ne soit pas toujours très explicite, on constate que les foyers égéens se présentent essentiellement sous trois formes : à plat, creusés et surélevés. Dans tous les cas, ils peuvent être simples ou complétés par des aménagements supplémentaires (revêtement ou bordure). La documentation actuelle ne permet pas de distinguer une évolution chronologique très nette et très précise entre ces différents types. On peut seulement constater que le type «à plat» est le plus couramment rencontré au Néolithique et à l'Age du Bronze. Les foyers creusés semblent, eux, plutôt employés au Néolithique alors que, inversement, l'usage des foyers surélevés semble se développer à l'Age du Bronze.

Les fours, quant à eux, sont essentiellement des fours en terre horizontaux. Ils sont surtout reconnus dans les Balkans (Bulgarie, Macédoine ex-yougoslave, Albanie) et le nord de la Grèce (Macédoine et Thessalie) du Néolithique à l'Age du Bronze. Les fours de pierre ou de tessons sont beaucoup moins souvent signalés et ne sont reconnus que sur quelques sites de Grèce centrale et du Péloponnèse. Les "fours-pithoi », tous datés de l'Age du Bronze, sont, pour leur part, caractéristiques du nord-est égéen (Chalcidique, Macédoine occidentale et Lesbos).

Les différents modes de fonctionnement décrits ci-dessus sont attestés par quelques traces archéologiques (résidus de combustion et rubéfaction de la surface des structures), mais ils ont surtout été suggérés par des observations ethnographiques et expérimentales. L'étude a montré que les foyers et les fours n'ont pas le même mode de fonctionnement, car ils sont destinés à des types de cuisson différents. Les autres fonctions évoquées sont secondaires et restent encore à confirmer du point de vue archéologique.

\section{BIBLIOGRAPHIE}

ASLANIS, I. 1985. Kastanas, Ausgrabungen in einem Siedlungshügel der Bronze - und Eisenzeit

Makedoniens 1975-1979. Die Frühbronzezeitlichen Funde und Befunde, Berlin.

BLEGEN, C. \& RAWSON, M. 1966. The palace of Nestor at Pylos in Western Messenia, I : The buildings and their contents, Princeton.

ARCQUe, P., touchais, G. \& TReUIL, R. 1992. Dikili Tash, dans Bulletin de Correspondance hellénique, 116, p.715-719.

Civilisations, 49 | 2009 
DelacretAZ, P. 1993. Les vieux fours à pain. Construire son four. Faire son pain, Yens-sur-Morges.

DEMARGNe, P. 1928. Etudes Crétoises, I, Paris.

DESHAYES, J. 1974. Fours néolithiques de Dikili Tash, dans Mélanges helléniques offerts à Georges Daux, p. 67-91.

EfSTRATiou, N. 1985. Agios Petros. A Neolithic site in the Northern Sporades, Oxford.

EVANS, A. 1921-1936. The palace of Minos at Knossos, I-IV et index, Londres.

GEORGIEV, G. 1963. Glavni rezultati ot razkopkite na Azmaskata selistna mogila prez 1961 g., dans Bulletin de l'Institut Archéologique Bulgare, 26, p. 157-174.

GEORGIEv, G. 1981. Die neolithische Siedlung bei Cavdar, Bezirk Sofia, Cultures préhistoriques en Bulgarie, dans Bulletin de l'Institut d'Archéologie Bulgare , XXXVI, p. 63-109.

GEORGIEV, G., NIKolov, V., NIKolova, V. \& COHADZIEV, S. 1986. Die neolithische Siedlung Kremenik bei Sapareva Banja, Bezirk Kjustendil, dans Studia Praehistorica, 8, Sofia.

GIMBUTAS, M. \& al. 1974. A Neolithic mound in Thessaly; preliminary report on 1973 and 1974

excavations, dans Journal of Archeology, 1, p. 277-302.

Gimbutas, M. Winn, S., \& ShimabuRu, D. 1989. Achilléion. A Neolithic Settlement in Thessaly, Greece, 6400-5600 BC, Los Angeles.

Goldman, H. 1932. Excavations at Eutresis in Beotia, Cambridge.

HÄNSEL, B. 1979. Ergebnisse der Grabungen bei Kastanas in Zentralmakedonien 1975-1978, JRGZM 26, p.167-202.

LAMB, W. 1936. Excavations at Thermi in Lesbos, Cambridge.

LEROI-GOURHAN, A. 1979. Séminaire sur les structures d'habitat. Témoins de combustion, dans Revista do museu Paulista, Nova serie, vol. XXVI, p. 7-57.

MOLIST, M. 1986. Les structures de combustion au Proche-Orient néolithique (10 000-3 700 BC), Thèse de Doctorat, Université Lyon 2.

PERLES, C., 1975. L'homme préhistorique et le feu, dans La Recherche, 60, p.829-839.

PREVOST, S. 1993. Les fours et les foyers domestiques en Egée au Néolithique et à l'Age du Bronze, DEA, Université de Paris I.

RENFREW, C., GIMBUTAS, M. \& ELSTER, E. 1986. Excavations at Sitagroi : a prehistoric village in Northeast Greece, I, Los Angeles.

RIDLEY, C. \& WARDLE, K. 1979. Rescue excavations at Servia 1971-1973 : a preliminary report, dans Annual of British School at Athens, 74, p. 185-230.

TREUIL, R. 1992. Dikili Tash (vol. I). Fouilles de J. DESHAYES, Paris.

SHAW, J. 1977. Kommos, rapport préliminaire, dans Hesperia 46.

TSOUNTAS, C. 1908. Les acropoles préhistoriques de Dimini et de Sesklo, Athènes.

VALMIN, N. 1938. The Swedish Messenia expedition, Lund.

WERNER, A. 1991. Reconstructions and experimental use of Late Neolithic bread ovens ", dans Archéologie expérimentale. Tome $1 \mathrm{Le}$ feu : le métal, la céramique. Actes du Colloque International «Expérimentation en Archéologie : Bilan et Perspectives », Paris. 


\section{NOTES}

1. J. Deshayes est le seul à s'être intéressé à l'étude morphologique de ces structures pour le site de Dikili Tash (Macédoine orientale).

2. Le Néolithique égéen est divisé en trois phases : Néolithique Ancien (7000-5700 BC environ), Néolithique Moyen (5700-4850 BC environ), Néolithique Récent (4850-3250 BC environ). L'Age du Bronze est aussi divisé en trois phases: Bronze Ancien (3250-1900 BC environ), Bronze Moyen (1900-1550 BC environ), Bronze Récent (1550-1050 BC environ). Il y a des nuances régionales; pour tous les Ages du Bronze, on parle de période helladique dans le Péloponnèse, de période minoenne en Crète et de période cycladique dans les îles.

3. C. Perles parle, elle, de «foyer-planı » dans Leroi-Gourhan, 1979, p.48; M. Molist (1986, p.57) préfère parler de "foyers posés", car ce sont les éléments de "tout un dispositif et pas seulement le feu lui-même qui ont été posés sur le sol».

4. On peut définir la terre à bâtir comme un mélange de terre plus ou moins argileuse, d'eau, de dégraissants d'origine végétale, minérale ou animale, employé comme matériau de construction pour confectionner du pisé, du torchis ou des briques. Voir la définition d'O. Aurenche, 1977, « terre à bâtir ».

5. MM I : Minoen Moyen I, c'est-à-dire environ 2100-1800 BC ; MM III : Minoen Moyen III, c'est-àdire environ 1700-1600 BC.

6. Surtout lorsque ce matériau contient des argiles de la famille de la kaolinite.

7. Dégraissants essentiellement minéraux qui ont pu être rajoutés volontairement à la terre argileuse qui en contient déjà naturellement.

8. Pour construire cette armature, d'autres végétaux ont pu être utilisés tels que des roseaux. Jusqu'à présent, ils n'ont pas été identifiés sur des vestiges de fours égéens.

9. Technique ne nécessitant pas obligatoirement de liant ni de moule.

10. Voir la partie sur le fonctionnement des fours (2.2).

11. Dans cette position, les pithoi présentent des similitudes avec les fours verticaux du ProcheOrient (tannours), mais les descriptions archéologiques trop sommaires ne permettent pas une analyse plus approfondie de cette ressemblance.

12. Les cendres et les charbons sont, comme dans le cas des foyers, les seuls produits de combustion retrouvés. Le bois, de toute taille et d'essences diverses, est sans doute le principal combustible.

13. Observations ethnographiques dans la région d'Emathie (en Grèce).

14. La cuisson des poteries se faisait sans doute à l'air libre, en tas.

\section{RÉSUMÉS}

Cette étude résume une synthèse récente sur les structures de combustion (foyers et fours) datant du Néolithique et de l'Age du Bronze en Grèce et dans le sud des Balkans. Elle présente les différents types de structures reconnus et leurs fonctionnements, ainsi que leurs différentes utilisations. Elle est le résultat d'une recherche bibliographique détaillée complétée par des observations ethnographiques et expérimentales. 
This study summarizes a recent synthesis about installations related to the use of fire (hearths and ovens) in the Neolithic and the Bronze Age periods in Greece and in the south of Balkans. It presents their varied types and functions as well as their different uses. It is the result of detailed bibliographic research complemented by ethnographic and experimental observations.

INDEX

Mots-clés : Fours, foyers, Grèce, néolithique, âge du bronze

Keywords : Ovens, hearths, Greece, neolithic, Bronze Age

\section{AUTEUR}

\section{SANDRA PREVOST-DERMARKAR}

Protohistoire Egéenne, Université Paris I, Malakoff, France 